\title{
Predictive Gene Signature for Pyrazolopyrimidine Derivative c-Src Inhibitor 10a Sensitivity in Melanoma Cells
}

\author{
Baris Kucukkaraduman, Can Turk, Anna L. Fallacara, Murat Isbilen, Kerem M. Senses, \\ Zeynep O. Ayyildiz, Muhammad W. Akbar, Michal Lotem, Maurizio Botta, and Ali O. Gure*
}

Cite This: ACS Med. Chem. Lett. 2020, 11, 928-932

Read Online

\section{ACCESS | Lill Metrics \& More | 回 Article Recommendations ｜（） Supporting Information}

ABSTRACT: Melanoma is a highly aggressive cancer with poor prognosis. Although more than $80 \%$ of melanomas harbor an activating mutation in genes within the MAPK pathway, which are mutually exclusive, usefulness of therapies targeting MAPK pathway are impeded by innate and/or acquired resistance in most patients. In this study, using melanoma cells, we report the efficacy of a recently developed pyrazolo $[3,4-d]$ pyrimidine derived c-Src inhibitor 10a and identify a molecular signature which is
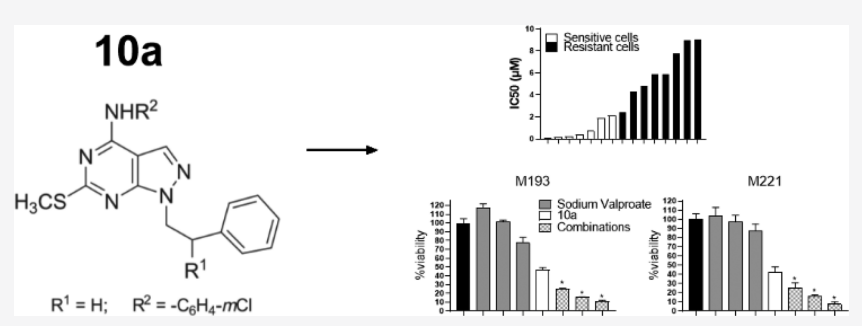
predictive of 10a chemosensitivity. We show that the expression of TMED7, PLOD2, XRCC5, and NSUN5 are candidate biomarkers for 10a sensitivity. Although an undifferentiated/mesenchymal/ invasive status of melanoma cells is associated with resistance to $10 \mathrm{a}$, we show here for the first time that melanoma cells can be sensitized to $10 \mathrm{a}$ via treatment with valproic acid, a histone deacetylase inhibitor.

KEYWORDS: melanoma, c-Src, drug resistance, valproic acid, sensitivity biomarkers

$\mathrm{M}$ elanoma is one of the most aggressive types of cancers with heterogeneous features. ${ }^{1}$ Treatment strategies and success rates vary depending on many factors, including feasibility of surgery, stage of the melanoma, presence of specific genetic mutations, and the rate of tumor growth. While surgery is the standard approach for localized and early stage melanoma, advanced stages require lymph node dissection, radiation therapy, and medication assisted therapies, including immunotherapy, chemotherapy, and targeted therapy.,3 However, long-term efficacy of such treatments is limited by their side effects and innate or acquired resistance. Therefore, new treatment strategies might help improve patient's quality of life and increase survival time.

The c-Src proto-oncogene is a nonreceptor protein kinase which is activated by different classes of cellular receptors, resulting in a diverse spectrum of biological activities including cell cycle progression, apoptosis, cell adhesion, migration, transformation, and various immune responses. ${ }^{4,5}$ Advanced stages of cancer, tumor growth, progression, and metastasis are associated with increased expression and activity of c-Src in melanoma and other solid tumors. ${ }^{4,6}$ Hence, c-Src represents a promising therapeutic target for the treatment of melanoma.

In this line, several Src inhibitors have been available for clinical assessment, including dasatinib and saracatinib, which are utilized in clinical trials in melanoma. Dasatinib is known to inhibit Src and other tyrosine kinases, including c-KIT, PDGFR, c-FMS, EPHA receptor, and BCR-ABL fusion. ${ }^{7}$ Nevertheless, a clinical trial has suggested that daily dasatinib treatment has only a minimal benefit in unresectable melanoma patients, the 6-month progression free survival (PFS) rate being only 13\% among these patients and with high rates of toxicity. ${ }^{8}$ Similarly, saracatinib was also minimally effective in a phase II study. ${ }^{9}$ Hence, there is a need for more potent c-Src inhibitors and/or a better definition of melanoma subtypes that would be suitable for Src inhibitor-based treatment protocols.

Pyrazolo $[3,4-d]$ pyrimidine derivatives show antitumor activity by inhibiting several serine/threonine or tyrosine kinases. ${ }^{10}$ $10 \mathrm{a}$ is the 6-methylthio derivative of pyrazolo[3,4-d]pyrimidine, which was shown to inhibit c-Src selectively. ${ }^{11}$ In this regard, we investigated the efficacy and molecular mechanisms of response to 10a in human melanoma cells. In this study, based on cytotoxic activity of 10a in 15 melanoma cells, we report the identification of a new gene signature which can predict chemosensitivity to 10a. Two distinct phenotypes of cells, defined as sensitive and resistant, were further analyzed to reveal an underlying mechanism for this differential response to $10 \mathrm{a}$. We found that proliferative or mesenchymal features of the cells are associated with distinct sensitivity of 10a. Through a protein-chemical interaction network analysis, we identified that three histone deacetylase

Special Issue: In Memory of Maurizio Botta: His
Vision of Medicinal Chemistry
Received: December 31, 2019
Accepted: February 18, 2020
Published: February 18, 2020


A

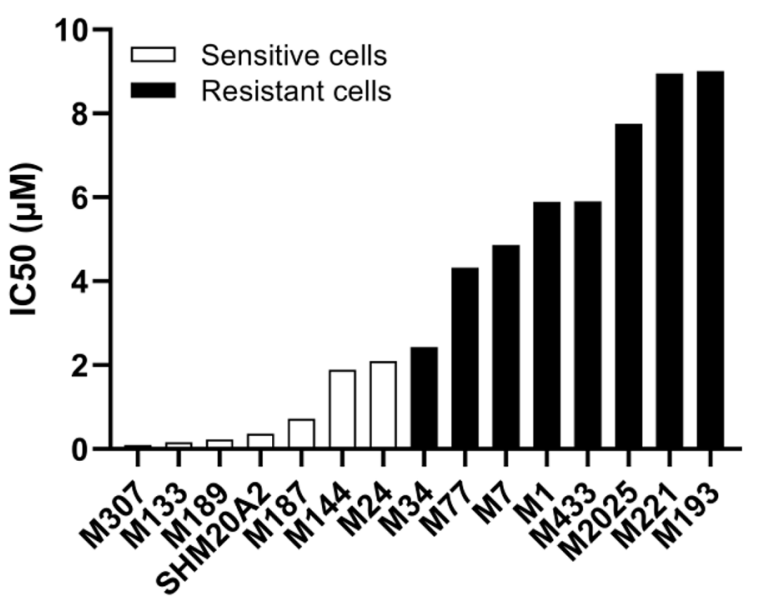

B

\begin{tabular}{|l|c|c|c|c|c|c|}
\hline Iterations & \multicolumn{2}{|c|}{$>900$} & \multicolumn{2}{c|}{$>800$} & \multicolumn{2}{c|}{$>700$} \\
\hline Direction & Same & Opposite & Same & Opposite & Same & Opposite \\
\hline \# of Genes & 9 & 0 & 24 & 0 & 46 & 0 \\
\hline
\end{tabular}

Figure 1. In vitro cytotoxicity analyses of c-Src inhibitor 10a on melanoma cells and hierarchical clustering of cell lines according to $10 \mathrm{a}$ chemosensitivity biomarker genes. (A) Fifteen melanoma cells were analyzed for sensitivity against $10 \mathrm{a}$. Cytotoxicity is expressed in terms of $\mathrm{IC}_{50}$ ( $y$-axis). (B) 10a chemosensitivity biomarker genes were determined by 2 -fold cross validation with 1000 iterations, and 9, 24, and 46 genes had a frequency of significance in more than 900, 800, and 700 iterations, respectively. (C) Melanoma cells were hierarchically clustered with 46 genes that were significantly correlated with cytotoxicity values against 10a for more than 700 iterations. Two clusters were defined as sensitive (M307, M133, M189, SHM20A2, M187, M144, M24) and resistant (M34, M77, M7, M1, M433, M2025, M221, M193). Standardized expression values were used for clustering, and clustering was performed with the complete linkage method on the heatmapper online tool. ${ }^{15}$

A

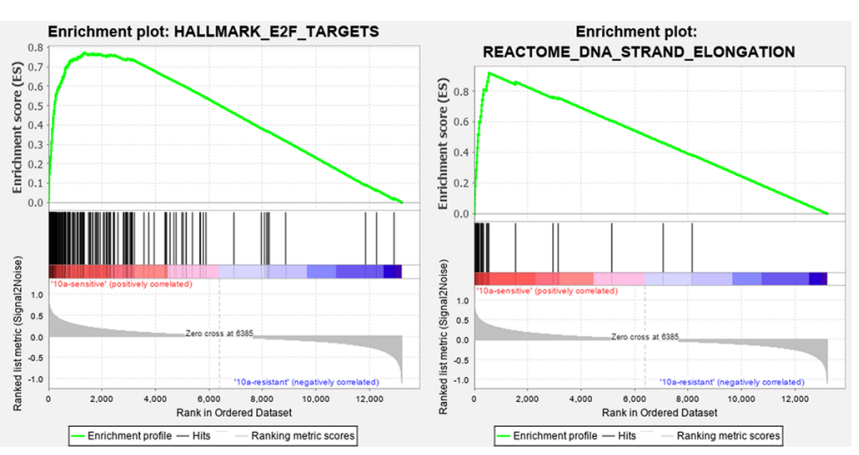

B

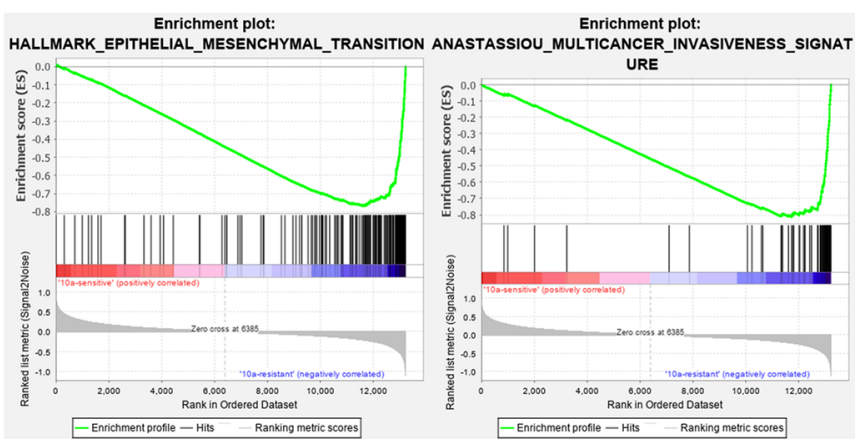

Figure 2. Comparative analysis of differentially enriched molecular gene sets among sensitive and resistant cells by GSEA. (A) Cells were grouped as sensitive and resistant based on clustering with the 10a chemosensitivity associated gene list seen in Figure 1C. Gene sets including cell cycle related targets of E2F transcription factor (left) and DNA strand elongation related genes (right) were enriched in 10a-sensitive melanoma cells. (B) Gene sets defining epithelial to mesenchymal transition as in wound healing, fibrosis, and metastasis (left) and multicancer invasion associated genes (right) showed enrichment in 10a-resistant melanoma cells. FDR values for each enrichment plot were less than 0.001 .

inhibitors, valproic acid, entinostat, and trichostatin A, were predicted to synergize with 10a. The synergizing effect of valproic acid was validated in vitro. We also aimed to define a minimal number of genes that could be used as biomarkers of 10a sensitivity. We show that the expression level of four genes can be used to predict drug sensitivity against 10a.

When tested on 15 melanoma cells, cytotoxic responses to $10 \mathrm{a}$ occurred over a remarkably wide range of doses with $\mathrm{IC}_{50}$ values ranging from 0.09 to $9.01 \mu \mathrm{M}$ (Figure 1A, Table S1). This variation strongly suggests underlying molecular differ- ences among these cells and thus prompted us to identify a gene signature of chemosensitivity for 10a. For this purpose, we correlated gene expression data from melanoma cells obtained by $\mathrm{C}$-MAP analysis with $\mathrm{IC}_{50}$ values using a 2 -fold cross validation with a 1000 iteration method (see Supporting Information). ${ }^{12}$ Briefly, in each iteration, cells were randomly divided into two groups, and a Pearson's $r$ value between 10a $\mathrm{IC}_{50}$ and gene expression data for each gene was calculated for these groups. This was repeated 1000 times. We then determined whether the correlations obtained for a given 
gene were consistently in the same direction (either positively or negatively correlated in both groups) where the results were statistically significant in all groups. This analysis showed that expression of 9 genes was consistently and significantly correlated with $\mathrm{IC}_{50}$ values in more than 900 iterations in the same direction. In more than 800 iterations, expression levels of 24 genes showed consistent and significant correlations with $\mathrm{IC}_{50}$ values in the same directions. We chose 46 genes whose expression profile showed significant concordance with chemosensitivity of $10 \mathrm{a}$ in more than 700 iterations for classifying melanoma cells (Figure 1B). Hierarchical clustering analysis with these 46 genes revealed two subgroups of cells which are relatively sensitive and resistant to $10 \mathrm{a}$ (Figure $1 \mathrm{C}$ ).

To investigate molecular mechanisms underlying the differential response to $10 \mathrm{a}$, we performed gene set enrichment analysis (GSEA) ${ }^{13}$ with sensitive and resistant subgroups defined with the 46-gene list. M307, M133, M189, SHM20A2, M187, M144, and M24 cells constituted the sensitive subgroup while M34, M77, M7, M1, M433, M2025, M221, and M193 constituted the resistant subgroup. GSEA revealed a significant enrichment for gene sets, including E2F target genes related to cell cycle and DNA strand elongation related genes in the sensitive subgroup, suggesting a proliferative phenotype in these cells (Figure 2A, Table S2). On the other hand, resistant subgroups showed significant enrichments of gene sets related to epithelial-mesenchymal transition and multicancer invasiveness signatures, indicating an invasive/mesenchymal phenotype in resistant cells (Figure 2B). These results are concordant with previous findings where melanoma cells were classified into invasive, proliferative, and intermediate categories. $^{14}$

To further investigate mechanisms behind resistance to 10a, we performed protein-chemical interaction network analysis with 46 genes using the NetworkAnalyst ${ }^{16}$ online tool. This revealed that, out of 18 resistance associated proteins, 15 extensively interact with a pyrazolopyrimidine derivative and three histone deacetylase (HDAC) inhibitors, valproic acid, trichostatin A, and entinostat (Figure 3). Expectedly, our analysis revealed (6-(4-(2-piperidin-1-ylethoxy)phenyl))-3pyridin-4-ylpyrazolo(1,5-a)pyrimidine, as a hub chemical, which is structurally similar to pyrazolopyrimidine derivative $10 \mathrm{a}$, showing interations with 12 resistance related proteins. Interestingly, three HDAC inhibitors have interactions mostly with resistance-associated genes, and are also known as differentiating agents. ${ }^{17,18}$ Valproic acid was a hub chemical with the highest number of interactions. Because our previous findings validated proliferative and invasive phenotypes in chemosensitive and resistant cells, respectively, protein chemical network analysis prompted us to use valproic acid to induce differentiation to enhance sensitivity of resistant cells to 10 a.

For this purpose, the 10a resistant M221 and M193 cells were treated with various doses of valproic acid (as sodium valproate) alone or in combination with $10 \mathrm{a}$ after $24 \mathrm{~h}$ of cell seeding. Cell viability was assessed $72 \mathrm{~h}$ after treatments. In M221 cells, $20 \mu \mathrm{M} 10 \mathrm{a}$ treatment resulted in a 55\% reduction in viability, whereas little or no decrease in viability was observed with 50, 200, and $800 \mathrm{nM}$ of sodium valproate treatment alone. However, combining $50 \mathrm{nM}$ sodium valproate with $20 \mu \mathrm{M} 10 \mathrm{a}$ resulted in a $75 \%$ decrease in viability. This effect increased to $84 \%$ when $200 \mathrm{nM}$ sodium valproate was used. The most significant effect was observed at $20 \mu \mathrm{M} \mathrm{10a}$

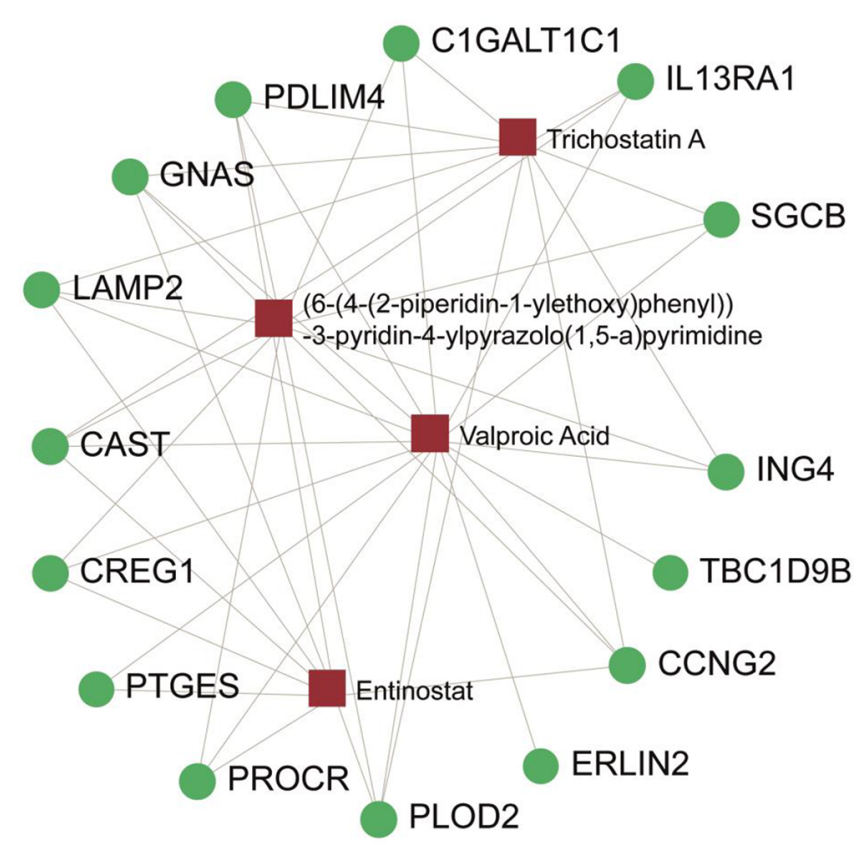

Figure 3. Protein-chemical interaction network with chemosensitivity associated genes. A protein-chemical interaction network was constructed using the NetworkAnalyst online tool to identify chemicals that interact with 10a-sensitivity associated proteins. Red squares represent the chemicals; green nodes represent proteins interacting with chemicals, and edges connect proteins to interacting chemicals. Betweenness filter (cutoff $=11.0$ ) was applied to obtain hub chemicals. Valproic acid interacts with 15 proteins represented in the network, while (6-(4-(2-piperidin-1-ylethoxy)phenyl))-3-pyridin4-ylpyrazolo(1,5-a)pyrimidine interacts with 12 proteins, trichostatin A interacts with 10, and entinostat interacts with 9.

and $0.8 \mathrm{mM}$ sodium valproate treatment, where a $92 \%$ decrease in cell viability was seen (Figure 4A). To quantify the degree of synergism, the combination index (CI) was calculated by the Chou-Talalay method in which $\mathrm{CI}<1$ shows synergistic effects, $\mathrm{CI}=1$ shows additive effects, and $\mathrm{CI}$ $>1$ shows antagonistic effects. ${ }^{19}$ Combined $10 \mathrm{a}$ and sodium valproate treatment showed synergistic effects for all combinations with $\mathrm{CI}$ values ranging from 0.766 to 0.603 (Figure 4A). Similarly, the sodium valproate-10a combination showed synergy in the more resistant M193 cells as well (Figure 4B). Twenty micromolar 10a treatment alone, decreased viability to $47 \%$ in M193 cells, whereas 0.8 , 4, and $6 \mathrm{mM}$ of sodium valproate showed little or no decrease in viability. Adding $0.8 \mathrm{mM}$ sodium valproate to $20 \mu \mathrm{M}$ of $10 \mathrm{a}$ resulted in a $75 \%$ decrease in viability. Combining $20 \mu \mathrm{M} 10 \mathrm{a}$ and $4 \mathrm{mM}$ sodium valproate treatment resulted in an $84 \%$ reduction in viability. Twenty micromolar 10a and $6 \mathrm{mM}$ sodium valproate combination resulted in $89 \%$ decrease in viability. CI values ranging from 0.835 to 0.698 suggested synergism in all combinations (Figure 4B). These results show that sodium valproate acts as a potent sensitizer for $10 \mathrm{a}$ in melanoma.

To identify a minimal number of biomarkers that could help classify melanoma subtypes which show differential 10a sensitivity, we selected 4 genes out of the 46 , based on highest fold-change $(\log 2 \mathrm{FC}>0.2$ and $<-0.2)$ in both directions and significance $(p$-value $<0.001)$ between sensitive and resistant cells (highlighted in Figure 1C). TMED7 and PLOD2, whose increased expression correlates with resistance to $10 \mathrm{a}$, along 
A

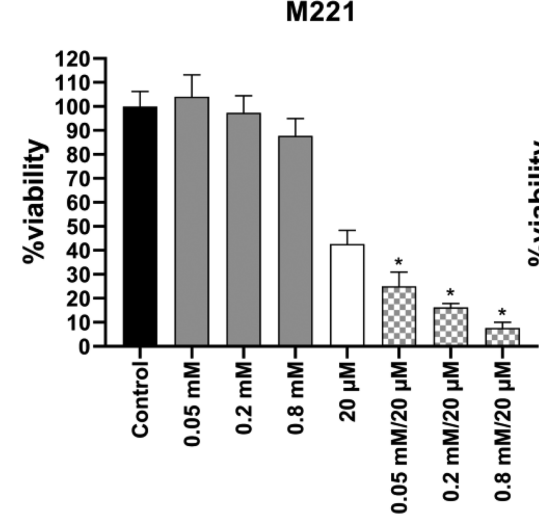

B

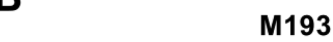

\begin{tabular}{lllll}
$\frac{\text { Dose }(\mu \mathrm{M})}{10 \mathrm{a}}$ & $\frac{\text { Dose }(\mathrm{mM})}{\text { Sodium Valproate }}$ & $\underline{\text { Effect }}$ & $\frac{}{\text { Combination }}$ \\
\hline 20.0 & 0.05 & 0.753 & 0.766 \\
20.0 & 0.2 & 0.839 & 0.681 \\
20.0 & 0.8 & 0.924 & 0.603
\end{tabular}

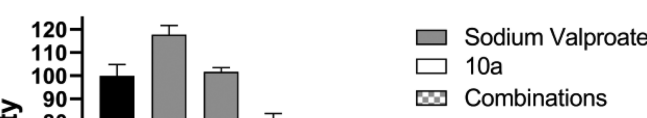

Combinations

Figure 4. Combination treatment of 10a-resistant M221 and M193 melanoma cells with 10a and sodium valproate. (A) After 24 h of cell seeding, M221 cells were treated with different doses $(0.05-0.8 \mathrm{mM})$ of sodium valproate in the absence or presence of 10a. Viable cells were quantified using Cell-Titer Glo assay after $72 \mathrm{~h}$. Combination index values of these combinations suggests synergistic effects in all combinations where CI $<1$ indicates synergy. (B) After $24 \mathrm{~h}$ of seeding, M193 cells were treated with different doses $(0.8-6 \mathrm{mM})$ of sodium valproate in the absence or presence of 10a. Viable cells were quantified using Cell-Titer Glo assay after $72 \mathrm{~h}$. Combination index values of these combinations suggests synergistic effects in all combinations. ${ }^{*} p<0.05$ ( $t$ test).

with XRCC5 and NSUN5 whose increased expression is indicative of sensitivity were chosen to be validated in vitro. Expression of these four genes were quantified by RT-qPCR analysis in four sensitive cells (M307, M189, SHM20A2 and M24) and five resistant cells (M34, M433, M2025, M221, and M193). Quantification of gene expression levels of four genes revealed that TMED7 and PLOD2 were upregulated in 10aresistant cells, whereas XRCC5 and NSUN5 were relatively downregulated, as expected (Figure 5).
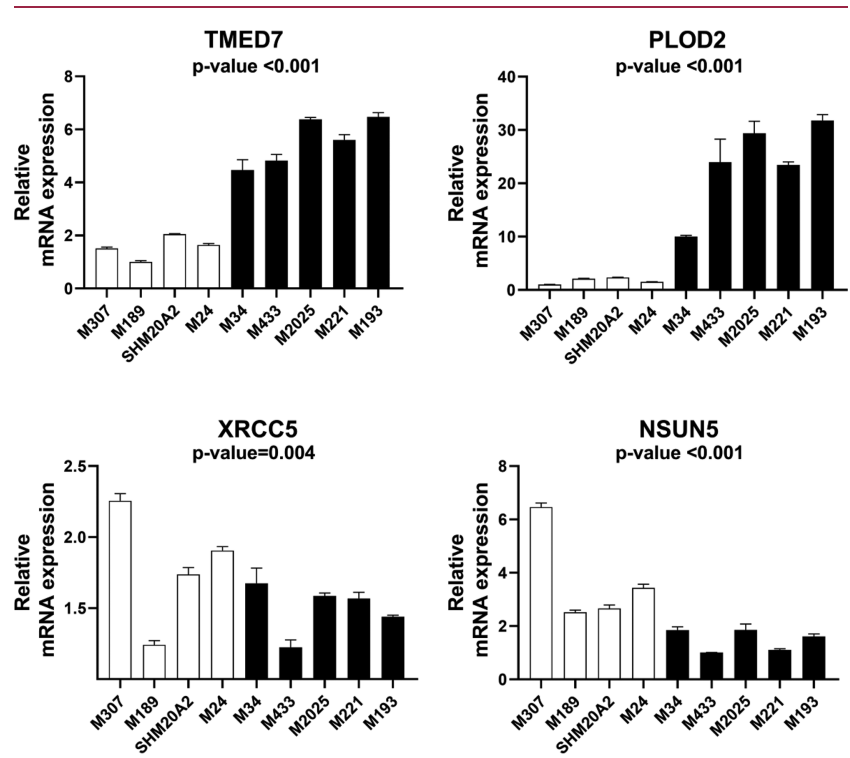

Figure 5. Gene expression analysis of candidate biomarkers for 10a chemosensitivity. TMED7, PLOD2, XRCC5, and NSUN5 expressions are quantified by real-time qPCR. GAPDH is used as endogenous control. M307, M189, SHM20A2, and M24 represent sensitive cells and M34, M433, M2025, M221, and M193 are 10aresistant cells. $p$-Values indicate $t$ test comparison of expression levels between sensitive and resistant cells.
The in vitro and in silico data we report here indicate that melanoma cells with proliferative or mesenchymal features clearly exhibit distinct sensitivity patterns for the c-Src inhibitor 10a. This is in agreement with our previous work, where a proliferative or invasive phenotype corresponded to 10a-sensitive and resistant cells, respectively. ${ }^{14}$ Additionally, combination of $10 \mathrm{a}$ with the histone deacetylase inhibitor, valproic acid, indicates that valproic acid can be used to enhance 10a cytotoxicity in these cells. Our expression data show TMED7, PLOD2, XRCC5, and NSUN5 are capable of classifying in vitro responses to 10a (Figure S1). Further studies of 10a in vivo, combination treatments with valproic acid, and clinical validation of biomarker genes would yield further insights into mechanisms of drug action, degree of cotreatment synergy, and clinical reliability of sensitivity biomarkers.

\section{ASSOCIATED CONTENT}

\section{Supporting Information}

The Supporting Information is available free of charge at https://pubs.acs.org/doi/10.1021/acsmedchemlett.9b00679.

Experimental details for cell culture, bioinformatics analyses, in vitro biological assays, combination index analysis, quantification of gene expression levels, hierarchical clustering with in vitro data and tables containing in silico data (PDF)

\section{AUTHOR INFORMATION}

\section{Corresponding Author}

Ali O. Gure - Department of Molecular Biology and Genetics, Bilkent University, Ankara 06800, Turkey; @orcid.org/00000002-4064-8608; Email: agure@bilkent.edu.tr

\section{Authors}

Baris Kucukkaraduman - Department of Molecular Biology and Genetics, Bilkent University, Ankara 06800, Turkey 
Can Turk - Faculty of Medicine, Department of Medical Microbiology, Lokman Hekim University, Ankara 06510, Turkey

Anna L. Fallacara - Department of Biotechnology, Chemistry and Pharmacy, "Department of Excellence 2018-2022", University of Siena, Siena 53100, Italy

Murat Isbilen - DNAFect Genetics Consulting R\&D and Biotechnology Inc., Kocaeli 41470, Turkey

Kerem M. Senses - Department of Molecular Biology and Genetics, Zonguldak Bulent Ecevit University, Zonguldak 67100, Turkey

Zeynep O. Ayyildiz - Department of Genome Sciences and Molecular Biotechnology, Izmir International Biomedicine and Genome Institute, Dokuz Eylul University, Izmir 35340, Turkey

Muhammad W. Akbar - Department of Molecular Biology and Genetics, Bilkent University, Ankara 06800, Turkey

Michal Lotem - Sharett Institute of Oncology, Hadassah Hebrew University Hospital, Jerusalem 91120, Israel

Maurizio Botta - Department of Biotechnology, Chemistry and Pharmacy, "Department of Excellence 2018-2022", University of Siena, Siena 53100, Italy; 이이.org/0000-0003-04566995

Complete contact information is available at:

https://pubs.acs.org/10.1021/acsmedchemlett.9b00679

\section{Notes}

OM.B.: Deceased on August 2, 2019.

The authors declare no competing financial interest.

\section{ACKNOWLEDGMENTS}

B.K. was funded by the TUBITAK-BIDEB 2211 scholarship program. This work was supported by the Scientific and Technological Research Council of Turkey (112S023) and COST action CM1106.

\section{ABBREVIATIONS}

MAPK, mitogen-activated protein kinase; TMED7, transmembrane P24 trafficking protein 7; PLOD2, procollagenlysine,2-oxoglutarate 5-dioxygenase 2; XRCC5, X-ray repair cross complementing 5; NSUN5, NOP2/Sun RNA methyltransferase 5; PDGFR, platelet-derived growth factor receptor; EPHA, ephrin A; C-MAP, connectivity map; GSEA, gene set enrichment analysis

\section{REFERENCES}

(1) Cancer Genome Atlas, N. Genomic Classification of Cutaneous Melanoma. Cell 2015, 161 (7), 1681-96.

(2) Strojan, P. Role of radiotherapy in melanoma management. Radiol. Oncol. 2010, 44 (1), 1-12.

(3) Domingues, B.; Lopes, J. M.; Soares, P.; Populo, H. Melanoma treatment in review. ImmunoTargets Ther. 2018, 7, 35-49.

(4) Irby, R. B.; Yeatman, T. J. Role of Src expression and activation in human cancer. Oncogene 2000, 19 (49), 5636-42.

(5) Shukla, D.; Meng, Y.; Roux, B.; Pande, V. S. Activation pathway of Src kinase reveals intermediate states as targets for drug design. Nat. Commun. 2014, 5, 3397.

(6) Lee, J. H.; Pyon, J. K.; Kim, D. W.; Lee, S. H.; Nam, H. S.; Kim, C. H.; Kang, S. G.; Lee, Y. J.; Park, M. Y.; Jeong, D. J.; Cho, M. K. Elevated c-Src and c-Yes expression in malignant skin cancers. J. Exp. Clin. Cancer Res. 2010, 29, 116.

(7) Araujo, J.; Logothetis, C. Dasatinib: a potent SRC inhibitor in clinical development for the treatment of solid tumors. Cancer Treat. Rev. 2010, 36 (6), 492-500.
(8) Kluger, H. M.; Dudek, A. Z.; McCann, C.; Ritacco, J.; Southard, N.; Jilaveanu, L. B.; Molinaro, A.; Sznol, M. A phase 2 trial of dasatinib in advanced melanoma. Cancer 2011, 117 (10), 2202-8.

(9) Gangadhar, T. C.; Clark, J. I.; Karrison, T.; Gajewski, T. F. Phase II study of the Src kinase inhibitor saracatinib (AZD0530) in metastatic melanoma. Invest. New Drugs 2013, 31 (3), 769-73.

(10) Schenone, S.; Radi, M.; Musumeci, F.; Brullo, C.; Botta, M. Biologically driven synthesis of pyrazolo[3,4-d]pyrimidines as protein kinase inhibitors: an old scaffold as a new tool for medicinal chemistry and chemical biology studies. Chem. Rev. 2014, 114 (14), 7189-238.

(11) Radi, M.; Brullo, C.; Crespan, E.; Tintori, C.; Musumeci, F.; Biava, M.; Schenone, S.; Dreassi, E.; Zamperini, C.; Maga, G.; Pagano, D.; Angelucci, A.; Bologna, M.; Botta, M. Identification of potent cSrc inhibitors strongly affecting the proliferation of human neuroblastoma cells. Bioorg. Med. Chem. Lett. 2011, 21 (19), 5928-33.

(12) Lotem, M.; Merims, S.; Frank, S.; Hamburger, T.; Nissan, A.; Kadouri, L.; Cohen, J.; Straussman, R.; Eisenberg, G.; Frankenburg, S.; Carmon, E.; Alaiyan, B.; Shneibaum, S.; Ozge Ayyildiz, Z.; Isbilen, M.; Mert Senses, K.; Ron, I.; Steinberg, H.; Smith, Y.; Shiloni, E.; Gure, A. O.; Peretz, T. Adjuvant Autologous Melanoma Vaccine for Macroscopic Stage III Disease: Survival, Biomarkers, and Improved Response to CTLA-4 Blockade. J. Immunol. Res. 2016, 2016, 8121985.

(13) Subramanian, A.; Tamayo, P.; Mootha, V. K.; Mukherjee, S.; Ebert, B. L.; Gillette, M. A.; Paulovich, A.; Pomeroy, S. L.; Golub, T. R.; Lander, E. S.; Mesirov, J. P. Gene set enrichment analysis: a knowledge-based approach for interpreting genome-wide expression profiles. Proc. Natl. Acad. Sci. U. S. A. 2005, 102 (43), 15545-50.

(14) Senses, K. M.; Ghasemi, M.; Akbar, M. W.; Isbilen, M.; Fallacara, A. L.; Frankenburg, S.; Schenone, S.; Lotem, M.; Botta, M.; Gure, A. O. Phenotype-based variation as a biomarker of sensitivity to molecularly targeted therapy in melanoma. MedChem Comm 2017, 8 (1), 88-95.

(15) Babicki, S.; Arndt, D.; Marcu, A.; Liang, Y.; Grant, J. R.; Maciejewski, A.; Wishart, D. S. Heatmapper: web-enabled heat mapping for all. Nucleic Acids Res. 2016, 44 (W1), W147-53.

(16) Zhou, G.; Soufan, O.; Ewald, J.; Hancock, R. E. W.; Basu, N.; Xia, J. NetworkAnalyst 3.0: a visual analytics platform for comprehensive gene expression profiling and meta-analysis. Nucleic Acids Res. 2019, 47 (W1), W234-W241.

(17) Gottlicher, M.; Minucci, S.; Zhu, P.; Kramer, O. H.; Schimpf, A.; Giavara, S.; Sleeman, J. P.; Lo Coco, F.; Nervi, C.; Pelicci, P. G.; Heinzel, T. Valproic acid defines a novel class of HDAC inhibitors inducing differentiation of transformed cells. EMBO J. 2001, 20 (24), 6969-78.

(18) Marks, P. A.; Richon, V. M.; Rifkind, R. A. Histone deacetylase inhibitors: inducers of differentiation or apoptosis of transformed cells. J. Natl. Cancer Inst 2000, 92 (15), 1210-6.

(19) Chou, T. C.; Talalay, P. Quantitative analysis of dose-effect relationships: the combined effects of multiple drugs or enzyme inhibitors. Adv. Enzyme Regul. 1984, 22, 27-55. 